\title{
Renouveau ethnique et renouveau religieux dans les « démocraties providentielles »
}

Dominique Schnapper

\section{(2) OpenEdition \\ 1 Journals}

Édition électronique

URL : http://journals.openedition.org/assr/3252

DOI : 10.4000/assr.3252

ISSN : $1777-5825$

Éditeur

Éditions de l'EHESS

Édition imprimée

Date de publication : 1 décembre 2005

Pagination : 9-26

ISBN : 2-7132-2045-9

ISSN : 0335-5985

Référence électronique

Dominique Schnapper, «Renouveau ethnique et renouveau religieux dans les « démocraties providentielles » », Archives de sciences sociales des religions [En ligne], 131-132 | juillet - décembre 2005, mis en ligne le 30 juin 2008, consulté le 20 avril 2019. URL : http://journals.openedition.org/ assr/3252; DOl : 10.4000/assr.3252

Ce document a été généré automatiquement le 20 avril 2019

() Archives de sciences sociales des religions 


\title{
Renouveau ethnique et renouveau religieux dans les « démocraties providentielles"
}

\author{
Dominique Schnapper
}

1 La conception de la société moderne comme tendanciellement bureaucratique, apaisée et rationnelle a été radicalement remise en question par l'observation des révolutions et des guerres $\mathrm{du} \mathrm{XX}^{\mathrm{e}}$ siècle. Le débat sur la part de la rationalité et la part des passions politiques ou des émotions ethnico-religieuses dans les sociétés démocratiques modernes reste ouvert. Nul autant que Shmuel Eisenstadt n'a contribué au développement de cette réflexion et l'on ne saurait lui rendre un meilleur hommage qu'en participant à cette interrogation, ce que je voudrais faire à partir de mon travail sur le politique et sur le principe de la citoyenneté qui organise la modernité politique ${ }^{1}$.

2 Dans un article qui date déjà de dix ans, j'avais présenté les théories des sociologues qui entendaient rendre compte du renouveau ethnique (ethnic revival) comme du renouveau religieux (religious revival) dans les sociétés démocratiques modernes, en les regroupant en théories de la «survivance » et théories de la «compensation " ${ }^{2}$. Pour les premiers auteurs, dont la pensée a longtemps dominé le champ sociologique, les sociétés modernes se caractérisaient par un processus de rationalisation, parallèle au rétrécissement du domaine du religieux et à l'affaiblissement de l'influence du religieux sur la vie collective. De même, les affiliations ethniques s'épuisaient progressivement avec le processus de construction des nations (nation building). De manière plus ou moins claire était avancée l'idée selon laquelle les pratiques religieuses et les affiliations ethniques étaient incompatibles avec la société moderne entraînée par le processus de rationalisation. Les penseurs influencés par le marxisme comme les théoriciens américains de l'assimilation, les sociologues du développement économique comme ceux de la construction des nations, à partir de prémisses ou d'intérêts différents, convergeaient pour voir dans les phénomènes religieux et ethniques essentiellement des survivances. Pour résumer leurs conclusions, on peut rappeler cette formule: «Ethnicity is one of the forms of 
Gemeinschaft that has survived (souligné par moi) in a rationalized, bureaucratized society " ${ }^{3}$. À ces théories de la survivance j'avais opposé les théories, plus récentes, qu'on pouvait regrouper dans les termes de la compensation à l'ambition moderne de rationalisation. Constatant le double renouveau de l'ethnique et du religieux, les sociologues de la compensation prolongeaient l'inspiration de Max Weber, qui avait montré l'irrationalité structurelle du monde «désenchanté» par l'ambition de rationalité. Loin d'être de simples survivances, les renouveaux religieux et ethniques pouvaient être analysés comme des produits de la modernité elle-même, liés au besoin éternel des hommes de donner un sens à leur destin face au mal et au malheur.

3 Je voudrais reprendre ces analyses à la lumière de la connaissance et de la réflexion accumulées depuis dix ans. Il m'apparait aujourd'hui plus clairement que les théories de la survivance, qui impliquent l'affaiblissement programmé des identifications ethnicoreligieuses ${ }^{4}$ sont, de toute évidence, insuffisantes. D'une part, on ne peut que constater que ces identifications et les passions qu'elles suscitent, loin de s'épuiser avec l'extension de la société technicienne et de l'idée démocratique, semblent au contraire se multiplier et s'affirmer de manière plus éclatante. D'autre part, les mouvements de renouveau ne sont pas seulement portés par des personnes ou des mouvements sociaux pré-modernes ou marginaux mais, le plus souvent, par les membres des catégories sociales qui participent activement aux processus économiques et politiques caractéristiques de la modernité. Les théories de la compensation paraissent donc plus fécondes, en ce qu'elles montrent bien que les mouvements de renouveaux ethniques et religieux sont le produit de la modernité elle-même.

4 Mais le terme de compensation me parait aujourd'hui trop connoté par la psychologie et, surtout, l'interprétation en termes de produit de la modernité exige une élaboration plus poussée. Je voudrais donc reprendre l'effort de compréhension de ce double renouveau, prolonger l'élaboration du lien entre la modernité et ces renouveaux, en l'inscrivant dans une analyse sociologique de la démocratisation contemporaine. Il s'agit de montrer comment la citoyenneté dite classique, en se transformant sous l'effet de la dynamique démocratique, tend à favoriser l'émergence des renouveaux ethniques et religieux - ce qui conduit à poser à nouveaux frais la question de l'avenir des démocraties.

5 La « citoyenneté » connaît une telle mode, politique et scientifique, que le terme a fini par perdre de son sens rigoureux, son extension de plus en plus large rendant sa compréhension de plus en plus faible. Je voudrais donc revenir sur ce qui me paraît l'essentiel dans ce qu'on appelle désormais la citoyenneté classique ${ }^{5}$.

6 La citoyenneté a un sens juridique - ensemble de droits et de devoirs -, mais c'est d'abord le principe de la légitimité politique. Les citoyens ne sont pas seulement des sujets de droit et de droits, chacun d'entre eux est détenteur d'une part de la souveraineté politique. C'est l'ensemble des citoyens, constitués en collectivité politique ou en " communauté des citoyens ", qui, par l'élection, choisit les gouvernants, puis contrôle et sanctionne leur action. C'est l'ensemble des citoyens qui est à la source du pouvoir et qui légitime que les décisions prises par les gouvernants soient exécutées. Les gouvernés reconnaissent qu'ils doivent obéir aux ordres des gouvernants parce que ceux qui leur donnent ces ordres ont été choisis par eux et restent sous leur contrôle. C'est le sens des expressions bien connues, le citoyen est « roi » ou le citoyen est « souverain ». La société des hommes démocratiques modernes s'est auto-constituée, elle ne tire plus sa légitimité que d'elle-même, elle n'admet plus aucune légitimité extérieure à elle-même. 
7 La citoyenneté est également la source du lien social ${ }^{6}$. Dans la société démocratique moderne, le lien entre les hommes n'est plus religieux ou dynastique, il est politique. Vivre ensemble, ce n'est plus partager la même religion ou être, ensemble, sujets du même monarque ou être soumis à la même autorité, c'est être citoyens de la même organisation politique. La société moderne démocratique se donne pour principe d'intégrer les populations par la citoyenneté en dépassant leurs diversités concrètes, en transcendant leurs particularismes.

8 C'est ce principe de transcendance des particularismes qui est au cœur de l'idée de citoyenneté. Constitués en "nation», pour reprendre l'article 3 de la Déclaration des droits de l'homme et du citoyen française de 1789 , les individus agissent dans l'espace public non en tant qu'individus concrets, avec leurs caractéristiques historiques et sociales particulières, mais en tant que citoyens. C'est pourquoi ils sont également citoyens, quels que soient leurs particularismes historiques ou religieux. Le nouveau principe de légitimité, proclamée en Amérique et en France à la fin du XVIII siècle, affirmait l'égalité civile, juridique et politique d'individus divers par leurs enracinements ethnico-religieux et inégaux par leurs conditions sociales. Malgré les préjugés de l'époque, en France, la citoyenneté fut étendue jusqu'aux juifs en septembre 1791, peu de temps après la proclamation du nouveau principe de légitimité. L'organisation politique reposait désormais sur une conception philosophique directement issue des Lumières, selon laquelle l'homme avait la capacité de s'arracher, au moins partiellement, à ses enracinements particuliers et d'entrer en communication avec les autres dans l'espace public commun à tous. Il pouvait cesser d'être le simple produit de son appartenance à un groupe réel. Son humanité était précisément définie par sa capacité de rompre avec les déterminations qui l'enfermaient dans une culture et un destin imposés par sa naissance, de se libérer des rôles prescrits.

9 Si la citoyenneté implique la transcendance des particularismes, elle est également fondée sur la souveraineté de l'individu-citoyen. C'est là une des tensions constitutives de l'ordre démocratique. La tension entre la transcendance des particularismes et celui de la souveraineté de l'individu peut être décomposée selon trois dimensions : tension entre le projet universel de la citoyenneté dans son principe ouvert à tous et la réalité des particularismes ; tension entre le contrôle social nécessaire et les émotions des hommes ; tension entre le principe de l'autonomie de l'individu et la participation des individus au collectif.

10 Le principe de la citoyenneté repose sur une double séparation historique : celle du politique et du religieux, celle de l'ethnique et du civique. Il se fonde sur l'autonomie croissante des divers domaines de la vie sociale. Pour être plus précis, il repose sur l'élaboration de l'ordre du civique comme mode de transcendance des affiliations et des passions indissolublement ethniques et religieuses, c'est-à-dire des identifications émotionnelles et partagées à des collectivités historiques ${ }^{7}$ particulières. L'ordre de la citoyenneté s'est établi contre les passions inclusives et exclusives liées aux identifications des individus à des collectivités historiques qui sont tout à la fois ethniques et religieuses, ou ethnico-religieuses.

11 La connaissance sociologique, à travers l'étude des relations interethniques, démontre les limites de ce processus de rationalisation des relations entre les hommes. L'objet même de l'étude - les relations interethniques - n'existerait pas si les sociétés démocratiques respectaient pleinement le principe de la citoyenneté dont elles se réclament. Or, l'histoire des démocraties révèle les manquements graves qui ont été apportés à ce 
principe. Il suffit d'évoquer ce qui a longtemps été perçu et vécu comme le "problème noir » aux États-Unis. Le souvenir de l'esclavage continue aujourd'hui encore à peser sur les relations entre ceux qui sont socialement perçus comme «Noirs" ou "AfricainsAméricains » et les autres Américains, alors même que l'universalité de la citoyenneté et l'égalité de leurs droits civiques furent affirmées après la Guerre civile. Le statut des juifs du 3 octobre 1940, en France, montre aussi comment les principes les mieux établis restent fragiles, on a pu exclure du corps des citoyens une partie de la population dans l'indifférence de la majorité de la population traumatisée par la défaite. La société coloniale en fournit un autre exemple. Elle était fondée sur l'inégalité des statuts juridiques des membres qui la composaient, alors que la légitimité de la démocratie moderne consiste à accorder l'égalité civile, juridique et politique à tous. C'est ainsi qu'on a pu voir naître cette monstruosité juridique par rapport au principe même de la citoyenneté : la nationalité sans la citoyenneté. En 1862, la Cour d'Alger affirmait, en effet, que « tout en n'étant pas citoyen, l'indigène est français ». Comme l'a remarqué un juriste : «La citoyenneté commune devrait entrainer normalement le suffrage universel et surtout le collège unique, aucune distinction ne pouvant être faite entre les citoyens pour l'exercice de leurs droits publics " ${ }^{8}$. On le sait, en Algérie, la pleine citoyenneté avec sa conséquence logique, le collège électoral unique, ne sera accordée qu'en 1958, à la veille de la disparition d'une société coloniale minée par ses contradictions.

12 En dehors même de ces manquements caractérisés dont l'histoire fournit des exemples nombreux, on ne peut que constater la persistance des identifications à l'intérieur des sociétés démocratiques les plus établies. La citoyenneté ne peut manquer de se conjuguer avec ces identifications. L'idée de la citoyenneté est certes fondée sur «le principe de l'égalité des êtres raisonnables et libres » selon la formule d'Eric Weil à propos du droit naturel, mais il est clair qu'elle n'est jamais réalisée en tant que telle. Ce serait confondre le principe ou la logique d'une organisation politique avec la réalité historique. La nation concrète est un particularisme. La participation à une société nationale est concrètement fondée sur toutes sortes d'éléments particuliers et particularisants, qu'on peut qualifier d'ethniques: la pratique d'une même langue (sauf cas exceptionnels), le partage par tous les nationaux d'une même culture et d'une mémoire historique singulière, la participation aux mêmes institutions, qu'il s'agisse de l'École ou de l'entreprise en passant par l'ensemble des pratiques strictement politiques. La familiarité immédiate qui s'établit entre les nationaux, quelles que soient par ailleurs les différences qui les séparent, est le produit de cette socialisation spécifique et de la vie commune à l'intérieur d'une société nationale concrète. Chaque individu est normalement attaché à ce qui constitue son univers familier à l'intérieur duquel il a élaboré son identité individuelle, liée à une identité collective. Chacun trouve sa nation avec ses particularismes à l'intérieur de soi, comme l'une des dimensions de sa propre identité.

13 L'abstraction et la rationalité de la citoyenneté ne peuvent, seules, organiser la société démocratique. Le principe civique par lui seul ne suffit pas pour faire vivre ensemble les hommes, qui ont besoin de partager une culture, des valeurs et des émotions pour « faire société ». Les sociétés démocratiques ne peuvent être seulement civiques, elles sont inévitablement à la fois ethniques - dans la mesure où elles partagent une histoire, réelle ou inventée, une culture et un projet communs - et civiques ${ }^{9}$. La singularité de la société organisée par le principe, les valeurs et les institutions de la citoyenneté par rapport aux autres modes d'organisation politique tient à ce que l'idée civique et le principe de la 
citoyenneté doivent en dernière analyse avoir la prééminence sur les particularismes ethniques ou religieux, sur les solidarités domestiques ou claniques.

L'insuffisance de la Raison pour organiser la vie des hommes a d'ailleurs été démontrée par l'histoire de la révolution française. Les révolutionnaires ont opposé au monde prémoderne une «Raison » non pas rationnelle et contrôlée, mais aussi passionnée que les passions religieuses qu'ils prétendaient dépasser. Le Peuple ou la Patrie a pris la place de Dieu comme objet d'une identification désormais vécue comme spontanée. Les passions des hommes suscitées au nom de la Raison n'ont pas été moins passionnées que leurs identifications ethnico-religieuses.

De plus, les passions ne sont pas nécessairement exclusives ou condamnables. Il est inévitable et peut-être même souhaitable d'aménager des valeurs holistes quand elles ne sont pas contradictoires avec les valeurs communes. Comme le dit Louis Dumont, l'art de la politique consiste aussi « à faire des entorses à notre droit, ou plutôt à y loger à titre secondaire des dispositions qui, en elles-mêmes, et si on ne prenait pas soin de limiter leur application, contrediraient son inspiration générale ${ }^{10}$. Et Todorov d'illustrer ce propos : «Revendiquer l'égalité de droit de tous les êtres humains n'implique nullement de renoncer à la hiérarchie des valeurs; chérir l'autonomie et la liberté des individus ne nous oblige pas à répudier toute solidarité ${ }^{11}$.

Doit-on dès lors penser que les conséquences de la citoyenneté sur les formes de la vie sociale sont limitées par les limites mêmes de la rationalité des hommes et que l'émergence des formes ethniques, religieuses ou ethnico-religieuses qu'on observe aujourd'hui traduit cette insuffisance de la rationalité pour répondre aux besoins d'émotions partagées et d'explication du malheur individuel et collectif qu'apportait le monde pré-moderne de l'ethnico-religieux?

Cette interprétation repose sur l'idée que seule la rationalité caractérise la société moderne. Si la citoyenneté ne relevait que de la rationalité, les théories de la survivance ou de la compensation rendraient effectivement pleinement compte de la persistance ou des renouveaux des affiliations ethniques et des pratiques et croyances religieuses. L'homme n'étant pas seulement un animal rationnel, ces renouveaux se développeraient, d'une part, parce que le passé ne disparaît jamais totalement et que les sociétés modernes ne sont que tendanciellement bureaucratiques et rationalisées (théories de la survivance) et, d'autre part, parce que la seule rationalité ne répond pas au besoin des hommes de trouver un sens au mal et au malheur; elle ne permet pas de répondre à l'interrogation métaphysique (théories de la compensation). Mais cette interprétation, si elle n'est pas fausse, me parait insuffisante, parce qu'elle laisse dans l'ombre le caractère utopique, ou prométhéen, de la citoyenneté.

18 La rationalité n'est en effet pas la seule caractéristique de la citoyenneté. Cette dernière comporte aussi et indissolublement une dimension utopique : c'est une utopie que de donner pour principe de légitimité et pour objectif à l'organisation sociale d'assurer la liberté et l'égalité juridique et politique d'individus divers et inégaux. Or, malgré ce principe ou cette utopie, il n'en reste pas moins que, comme toutes les sociétés humaines, les sociétés démocratiques imposent des contraintes qui, par définition, limitent la liberté de chacun ; elles restent également hiérarchiques et, en conséquence, limitent l'égalité de tous.

19 La citoyenneté est anti-naturelle, ses principes sont contredits par l'expérience sociale quotidienne. Chacun peut quotidiennement constater que, dans la vie concrète, « certains 
sont plus égaux que d'autres", selon la formule bien connue. L'égalité proclamée est souvent bafouée. Ne créerait-elle pas, en conséquence, du "refoulé », qui renforcerait les passions et les émotions liées aux identifications ethnico-religieuses? Le caractère utopique de la citoyenneté ne risque-t-il pas de causer plus de dégâts concrets que l'inégalité reconnue et institutionnalisée de l'ordre social pré-moderne? On reconnaîtra ici la transposition de la théorie du racisme proposée par Louis Dumont. Selon lui, la société moderne en proclamant l'égalité des conditions, en éliminant les différences de statut et en ne reconnaissant pas les inégalités de fait a pour effets pervers de faire naître le racisme. La hiérarchie inhérente à toute société étant refoulée par les principes proclamés de l'ordre social, elle risque toujours de revenir, comme tout refoulé, sous forme pathologique et monstrueuse, le racisme ${ }^{12}$.

Sans suivre cette analyse jusqu'à son terme, on peut avancer que le caractère utopique de la société des citoyens, allié au projet prométhéen de maîtriser le monde naturel grâce au progrès scientifique et technique, explique le refus de toutes limites qui caractérise la démocratie aujourd'hui. J'en évoquerai rapidement quelques illustrations: le refus des limites de la définition du corps politique, le refus des limites qu'on peut apporter au bien-être matériel ou moral des individus, la réticence à accepter la fin de l'âge de la procréation, à admettre le malheur, le vieillissement et la mort - ce qui définit la condition humaine.

21 La dynamique démocratique s'est d'abord exercée sur la définition du corps politique. On le sait, après avoir affirmé l'universalité du citoyen, les révolutionnaires français, en 1791, ont ensuite distingué les citoyens " actifs » des citoyens "passifs ", exclus des pratiques spécifiquement politiques : les pauvres, les indigènes des colonies, les femmes, les jeunes ne disposaient pas du droit de voter ni d'être élus. L'histoire du suffrage universel révèle la vocation potentiellement universelle de la citoyenneté. Ces catégories de la population se sont vues progressivement reconnaître les droits de la citoyenneté au nom des valeurs universelles proclamées lors de la Révolution: les pauvres et les indigènes, à la suite de l'abolition de l'esclavage, en 1848, les femmes en 1944, les jeunes entre 18 et 21 ans et les nouveaux naturalisés en 1974. C'est aussi au nom des principes universels que les colonisés ont justement revendiqué les droits de la citoyenneté que proclamaient les colonisateurs, mais qu'ils se réservaient à eux-mêmes. Il existe une poussée interne à la démocratie elle-même, qui tend, en fonction de ses propres valeurs, à étendre le corps politique ${ }^{13}$.

Aujourd'hui, alors que le suffrage dit universel semble établi, trois grands débats manifestent de l'impatience devant les limites juridiques qui sont apportées à la définition du corps politique. Le premier porte sur les enfants : à partir de quel âge devraient-ils être pleinement reconnus comme citoyens? En dessous de quel âge est-il légitime de continuer à ne pas leur accorder le droit de vote, alors qu'ils sont soumis aux contraintes de l'ordre politique ? Certains prônent de le faire à seize ans, voire plus tôt, dès qu'ils disposent d'une forme de responsabilité, civile, pénale ou financière. Le second débat s'exprime à travers les théories de ceux qui militent en faveur de la " citoyennetérésidence ». Selon eux, exclure des pratiques étroitement politiques (droit de vote et droit d'être élus) les non-nationaux, alors même qu'ils bénéficient de l'égalité des droits civils, économiques et sociaux, serait contraire aux valeurs démocratiques, puisque les étrangers, par leur présence même, leur « résidence », font, de fait, partie intégrante de la société nationale. En d'autres termes, imposer la limite de la nationalité à la citoyenneté est, selon eux, en tant que tel illégitime ; il faudrait accorder automatiquement les droits 
de la citoyenneté à tous ceux qui résident sur le sol national, même, selon certains auteurs, s'ils sont en situation irrégulière. Enfin, un dernier débat se développe à l'instigation des militants du mouvement international en faveur des droits politiques des animaux, peu représenté en France mais fort actif dans le monde anglophone, sous la forme du the great ape project ou projet des grands singes. Il serait conforme aux valeurs démocratiques, selon ces penseurs, que les grands singes (orangs-outangs, chimpanzés, gorilles), qui partagent avec les hommes la plus grande part de leur capital génétique et, surtout, qui subissent les conséquences des décisions politiques prises dans les sociétés humaines, se voient reconnaître des droits également politiques. Ils font d'ailleurs écho aux penseurs de l'écologie profonde (deep ecology) qui entendent donner des droits à la nature ${ }^{14}$. Les débats autour de ces théories illustrent l'affaiblissement de la frontière nette entre les êtres humains, dotés de responsabilité et de liberté, et les animaux, qu'avaient posée la tradition chrétienne et la pensée humaniste.

La poussée vers l'extension des limites du corps politique continue, on le voit, à s'exercer, manifestant la force de la dynamique démocratique. Un ouvrage récemment traduit au cours des dernières années dans la plupart des langues européennes - ce qui montre l'écho qu'il rencontre -, en donne d'ailleurs une illustration presque caricaturale: «Le nouveau projet de libération ne peut être qu'un projet de démocratie générale, qui étendra l'espace public, au-delà du champ politique traditionnel, à l'économique et au social. La démocratie générale implique l'abolition des formes de répartition inégale du pouvoir politique et économique, l'abolition des structures institutionnelles qui les reproduisent, et l'abolition des structures hiérarchiques au foyer, dans l'entreprise, à l'école et dans toute la société. Elle implique l'élimination des rapports de domination au niveau social et de l'idée de domination du monde naturel qu'ils induisent (...) La démocratie ainsi conçue est inconciliable avec toute forme d'inégalité dans la répartition du pouvoir, toute concentration du pouvoir politique, social ou économique. Elle est donc incompatible avec les rapports marchands et de propriété, qui concentrent inévitablement le pouvoir. Incompatible aussi avec les structures hiérarchiques, qui impliquent la domination, institutionnalisée (comme celle des femmes par les hommes) ou « objective » (comme celle du Sud par le Nord dans la division du travail instaurée par le marché), et avec la notion de maîtrise du monde naturel qu'elles induisent. Fondamentalement incompatible, enfin, avec tout système clos de croyances, de dogmes ou d'idées. La démocratie n'a donc rien à voir avec l'image qu'on en a aujourd'hui... » ${ }^{15}$. Il s'agit de prôner ce qu'on pourrait appeler la « démocratie totale ", celle qui ne connaît ni bornes ni frontières, qu'elles soient sociales, humaines ou géographiques.

Le développement des droits dits sociaux traduit aujourd'hui, tout particulièrement, l'ambition démocratique d'assurer l'égalité réelle de tous. Le développement de l'État providence, stabilisé depuis la fin de la Seconde Guerre mondiale dans l'Europe libérale, en est l'instrument. Le terme d'État providence ne doit pas être limité à son sens étroit ou classique, c'est-à-dire la protection des individus contre la maladie, la vieillesse, les charges de famille et le chômage, ainsi que l'assistance aux plus pauvres et aux plus marginaux. Ce premier État providence, qui fut progressivement établi depuis la moitié $\mathrm{du} \mathrm{XIX}^{\mathrm{e}}$ siècle et consolidé après la Seconde Guerre mondiale, n'est qu'une des dimensions de l'intervention collective. L'État d'aujourd'hui, qu'on peut appeler "providentiel ", est, beaucoup plus largement, chargé de pourvoir à la sécurité, à l'éducation, à la formation culturelle et sportive de tous et même de reconnaître les identités culturelles particulières. Il ne cesse d'étendre ses interventions pour répondre à 
tous les besoins des hommes. On connaît l'accroissement considérable des sommes consacrées à l'éducation par le système scolaire et universitaire au sens large (y compris les enseignements spécialisés ou professionnels et la formation permanente), la part croissante du budget qui est consacrée à la politique dite culturelle menée, en France ${ }^{16}$, par le ministère du même nom (qui tend à obtenir jusqu'à $1 \%$ du budget de l'État) et, de plus en plus, relayée et développée par les collectivités locales. Ce qu'on peut appeler l'État providence sportif n'est pas non plus négligeable, puisqu'en France les ministres visent à obtenir un budget égal à $0,5 \%$ du budget de l'État et que les pouvoirs locaux ne cessent d'organiser et de financer les pratiques sportives de tous niveaux, en recrutant des animateurs professionnalisés, des organisateurs diplômés et des fonctionnaires spécialisés. On pourrait également parler de l'État providence agricole ${ }^{17}$.

Les interventions se développent également dans le domaine qu'on peut qualifier d'identitaire ou d'ethnique. C'est ainsi que des politiques multiculturelles officielles ont été adoptées, par exemple au Canada et en Australie, deux pays d'immigration massive, qui consistent à transférer une part des ressources collectives pour assurer la persistance des cultures dites d'origine des migrants, grâce à l'activité de gestionnaires de l'ethnique : travailleurs sociaux, traducteurs, enseignants spécialisés à tous niveaux, animateurs d'associations culturelles et de médias ethniques. En France, le principe invoqué de l'universel républicain, selon lequel les catégories ethniques sont volontairement ignorées, s'oppose à ce qu'on adopte ce type de politique, mais, depuis deux ou trois ans, l'« ethnisation » des politiques publiques s'étend dans les faits, sous le masque de critères géographiques ou sociaux. C'est ainsi que se sont développées les politiques de la Ville successives, adoptées depuis vingt ans pour « redynamiser » les quartiers les plus pauvres où la population d'origine étrangère est majoritaire, et favoriser l'intégration de leurs habitants ; leur action est renforcée par les dispositions concernant les Zones d'Éducation Prioritaire (ZEP) en faveur d'établissements scolaires situés dans les mêmes quartiers. Dans tous les cas, cela consiste à donner des moyens complémentaires aux populations dont la migration est proche, même si l'on adopte des critères géographiques pour définir les bénéficiaires; ces politiques seraient qualifiées de «multiculturelles» dans d'autres pays. Il s'agit en effet d'inventer des catégories nouvelles qui restent définies en termes sociaux ou géographiques mais qui correspondent en fait grossièrement à des catégories ethniques à cause du regroupement spatial des migrants - ce que chacun sait -, pour les faire bénéficier du transfert d'une partie des ressources collectives, afin de favoriser l'égalité réelle de tous.

26 C'est la raison pour laquelle le déficit de l'État providence n'est pas seulement lié à la crise économique et financière, il est structurel. Les besoins de sécurité, de bien-être et d'égalité sont, par définition, indéfinis et se renouvellent lorsque se transforment les sociétés et que les exigences des individus, au fur et à mesure qu'elles sont satisfaites, se font plus pressantes. C'est ce que révèle l'invention continue de nouveaux métiers du bien-être pour répondre à ces besoins nouveaux qui s'étendent jusqu'aux animaux domestiques, devenus à leur tour les objets de soins médicalisés. L'État providence nourrit les insatisfactions, parce que la réponse tarde toujours par rapport aux demandes et que, même dans les sociétés riches, les ressources sont par définition limitées quand les besoins, eux, donc les demandes, sont illimités.

27 Cette volonté d'assurer non plus seulement l'égalité formelle mais l'égalité « réelle » pour reprendre l'opposition classique de la tradition marxiste entre la liberté et l'égalité « formelles » et la liberté et l'égalité « réelles »- a pour effet de particulariser l'action de 
l'État. Celui-ci définit inévitablement des catégories nouvelles chaque fois qu'il élabore une nouvelle politique. Aux demandes toujours accrues des individus, il répond par des mesures toujours plus particulières pour assurer plus de sécurité, plus de bien-être aux individus et plus d'égalité entre eux. Dès lors, les interventions se multiplient, deviennent de plus en plus précises, se superposent, se sédimentent et s'inscrivent dans les institutions d'une manière qui semble nécessaire et irréversible. Une partie de la société devient "providentielle », c'est-à-dire directement liée aux interventions de l'État providence, dont le nombre des agents se multiplie, et à son inévitable bureaucratisation.

Dernière illustration d'un refus des limites, touchant à la condition humaine elle-même. Le culte rendu au corps, à la vigueur, à la réussite sportive, à la jeunesse et à la santé, cette dernière haussée au rang de "valeur " première des sociétés démocratiques dans tous les sondages; les aspirations de certaines femmes à procréer au-delà de l'âge naturel ; le refus du malheur et de la misère qui impose dans la vie sociale l'utilisation d'euphémismes («non voyants» pour "aveugles», « handicapés» pour «infirmes », " personnes à taille réduite » pour « nains », « partir » pour « mourir », etc.) ; la difficulté à faire leur place aux plus vieux dans la vie sociale, souvent marginalisés dans des institutions spécialisées ; le scandale que constitue la mort, quel que soit l'âge auquel elle survient; tous ces traits constituent autant d'exemples de ce refus des limites de la condition humaine. La modernité démocratique se donne pour ambition de réaliser le projet prométhéen de contrôler la nature et de prolonger la durée de la vie humaine grâce aux réussites remarquables de la science et aux prouesses de la technique. La société démocratique est une société de performance.

29 Son utopie est non transcendantale. Elle porte sur les conditions de vie concrètes et immédiates des individus, sa logique est celle du hic et nunc. Dans la démocratie providentielle, la recherche du bien-être ou du mieux-être ${ }^{18}$ sans limites de chaque individu - son confort physique, moral, intellectuel et identitaire - tend à devenir l'objectif premier et la légitimité de l'organisation sociale. Les hommes démocratiques aspirent à conjuguer les contraires, la pleine liberté et la protection absolue ${ }^{19}$. Ils accordent la prééminence au concret immédiat aux dépens de toute forme de transcendance, sans accorder de légitimité à la tradition et à la transmission, sans se donner pour ambition de construire l'avenir au nom d'une Idée. L'homo democraticus n'entend mourir ni pour Dieu ni pour la Patrie.

30 «Si l'esprit de la grande majorité du genre humain se concentrait jamais dans la seule recherche des biens matériels, on peut s'attendre qu'il se ferait une réaction prodigieuse dans l'âme de quelques hommes... Je serais surpris si, chez un peuple uniquement préoccupé de son bien-être, le mysticisme ne faisait pas bientôt des progrès ${ }^{20}$. La positivité de l'utopie démocratique oppose en effet peu de résistance au développement des émotions des hommes et à leurs interrogations sur l'ultime.

La vocation sans limites du processus démocratique et les déceptions que nourrit inévitablement un projet tout à la fois utopique et concret suscitent le retour, sous de nouvelles formes, des passions, des émotions et des identifications dans l'ordre du religieux et de l'ethnique. On n'épuise pas le sens du renouveau des passions ethnicoreligieuses par les théories de la survivance, ce qui impliquerait qu'elles sont destinées à disparaître au fur et à mesure que la démocratie déploie ses conséquences sur les formes de la vie sociale. On n'en épuise pas non plus le sens par les théories de la compensation à la rationalité excessive de la société moderne, parce que celle-ci n'est pas seulement rationnelle, mais aussi utopique. On doit comprendre le renouveau des affiliations 
ethniques et les formes de retour du religieux à la fois à partir du processus de rationalisation et à partir de l'ambition utopique de la société démocratique qui est intimement et nécessairement lié à ce processus.

Le refus de toute limite, conjugué à l'exigence de résultats concrets et immédiats, nourrit inévitablement l'insatisfaction et la déception. L'homme démocratique ne peut pas ne pas constater que ses aspirations à jouir d'une sécurité et d'un bien-être matériel ou moral, toujours plus grands, rencontrent des limites. La passion de l'égalité ne pouvant jamais être pleinement satisfaite suscite chez les individus le sentiment de l'échec, l'envie, voire le ressentiment. Étant donné les ambiguïtés de la notion elle-même, soulignée par les philosophes depuis Aristote, la passion de l'égalité est impossible à satisfaire pleinement, d'autant plus que la prééminence accordée à l'individu aux dépens du collectif fait de la recherche de l'« authenticité » de chacun une valeur centrale. Tout ce qui caractérise l'individu en propre devient précieux et mérite d'être, en tant que tel, respecté. Mais l'aspiration à l'égalité couplée avec la recherche de l'authenticité, qui caractérise l'homo democraticus contemporain, ne tend-elle pas à miner le principe même de la transcendance des particularismes qui est au fondement de la citoyenneté ?

Les identifications religieuses et ethniques sont vécues comme spontanées et les institutions démocratiques ne peuvent que les reconnaître, même si elles doivent les contrôler pour que les identifications des uns ne menacent pas les identifications, également légitimes, des autres. L'ordre démocratique implique donc de manière nécessaire que des institutions religieuses et politiques organisent la transcendance des particularismes ethniques, religieux ou ethnico-religieux. Les institutions ecclésiales, d'un côté, les institutions politiques qui structurent la représentation (militantisme organisé, participation électorale, partis politiques, pratiques parlementaires, etc.), de l'autre, ont pour fonction de contrôler les identifications et les émotions ethnico-religieuses. Les tensions entre le contrôle rationnel par les institutions ecclésiales ou politiques et les émotions spontanées, entre les aspirations au bien-être de l'individu et les conditions de sa participation au collectif ont été en effet gérées ou régulées par les institutions de ce qu'on appelle dans la tradition française, la « République », c'est-à-dire les institutions de la citoyenneté, désormais qualifiée de classique. En France, les Républicains avaient directement imité le modèle de la régulation des croyances et des pratiques qu'assurait l'institution ecclésiale. La morale civique était empruntée au catéchisme, l'action des instituteurs remplaçait, en la prolongeant, celle du clergé proche du peuple, la hiérarchie de l'institution étatique était calquée sur celle de l'Église catholique. Avec le déploiement de la démocratie providentielle, l'individu devient premier, l'identification aux particularismes tend à primer sur l'aspiration à l'universel, l'expression des émotions est valorisée aux dépens du contrôle de soi, les institutions de l'Église et de la démocratie représentative sont affaiblies par la critique systématique ou radicale. L'individu-citoyen, parce qu'il est la source de la légitimité politique, refuse que la tradition fonde la légitimité des institutions ecclésiales et politiques. Il s'accorde à lui-même le droit absolu de critiquer toutes les institutions en tant que telles.

C'est ainsi qu'on voit naître les mouvements religieux et ethniques qui ont pour caractéristiques communes de refuser toute forme d'institutionnalisation et d'adopter les formes à la fois émotionnelles et syncrétiques - sorte de bazar des croyances et des identifications où chacun peut librement choisir de quoi faire son miel. C'est ce que des sociologues ont analysé sous le terme de Nouveaux Mouvements Religieux ${ }^{21}$. Lorsque la légitimité de la transmission est contestée dans son principe, les individus reconstruisent 
des liens qu'ils choisissent ou inventent, dans l'ordre religieux comme dans l'ordre familial ou politique. C'est encore son bien-être que l'homme démocratique recherche lorsqu'il élabore des modalités originales, individuelles et provisoires, du croire ou qu'il invente des identifications, également individuelles et provisoires, à des collectivités historiques, plus ou moins réelles ou imaginées. On peut célébrer cette invention de liens choisis librement par des individus libres et égaux ${ }^{22}$, mais c'est là une possibilité réservée à ceux qui sont susceptibles de mobiliser des ressources économiques, culturelles et sociales, bref qui ont effectivement la capacité de «créer du lien » à partir de leur individualisme. Cette possibilité est inégalement répartie. Étant donné les valeurs mêmes auxquelles se réfèrent les sociétés démocratiques, on ne saurait oublier, pour apprécier l'ordre qu'elles instituent, que les institutions fortes protègent les faibles.

Ces formes de référence à des croyances et à des collectivités historiques, vécues comme spontanées et immédiates, remettent en cause les institutions de la communauté des citoyens. Les aspirations démocratiques risquent de miner, sous l'effet de leur dynamique interne, son principe de légitimité. En reconnaissant toujours plus les particularismes ethnico-religieux dans le domaine public, en légitimant les émotions spontanées aux dépens du contrôle par les institutions, en remettant en question les institutions politiques et ecclésiales héritées du passé, les pratiques des hommes démocratiques affaiblissent les institutions communes et le contrôle qu'elles exerçaient sur l'expression des passions individuelles et collectives.

Or, le civique est un principe d'inclusion de tous, même si dans les faits il comporte toujours, et sans doute inévitablement, des limites et des manquements. En revanche, l'appartenance ethnico-religieuse est un principe d'exclusion des autres, même s'il peut être corrigé ou amendé dans les faits. Dans son principe même en effet, la société des citoyens est ouverte à tous ceux qui sont susceptibles de participer à la vie politique. La société organisée par la citoyenneté est, par définition, plus ouverte aux autres que les collectivités historiques, définies en termes religieux, culturels ou historiques. Toute organisation nationale prévoit que l'étranger puisse se voir reconnaitre le droit d'entrer dans la communauté politique, moyennant le respect d'un certain nombre de conditions que fixe l'État. On peut acquérir la nationalité française, suisse ou allemande par la naturalisation, même si les exigences imposées au candidat sont, dans chaque cas, différentes. En revanche, on appartient au " peuple corse " par sa naissance, on ne peut pas devenir corse. Mais si la Corse était constituée en nation souveraine, elle ne pourrait pas ne pas prévoir les modalités, juridiques et administratives, selon lesquelles certains individus pourraient acquérir la nationalité. Les rabbins rendent difficile l'entrée dans le peuple juif tel que le définit la Tradition religieuse, mais l'État d'Israël a admis comme citoyens israéliens un grand nombre d'individus qui ne sont pas juifs $(20 \%$ de la population) et d'autres qui se considèrent comme juifs, mais ne sont pas reconnus juifs par les tribunaux rabbiniques.

Jusqu'où peut-on reconnaitre institutionnellement les identités particulières sans remettre en cause l'existence de l'espace public commun, dans lequel des individus qui s'identifient à des collectivités historiques réunies dans la même société nationale, peuvent vivre ensemble grâce au respect des règles de la citoyenneté? Jusqu'où le multiculturalisme historique ou religieux de toute société nationale peut-il être reconnu dans l'espace public sans que les valeurs et le projet politique commun soient remis en cause? Selon la citoyenneté classique, la réponse est en théorie claire, même si elle ne permet pas de résoudre les problèmes pratiques qui se posent quotidiennement. Si les 
valeurs et les spécificités culturelles des groupes particuliers sont compatibles avec les exigences de la vie commune et les valeurs collectives - la liberté et l'égalité de toutes les personnes -, les citoyens et les étrangers régulièrement installés sur le sol national sont libres de cultiver leurs particularités dans leur vie personnelle comme dans la vie sociale, à condition de respecter les règles de l'ordre public. Ce droit est inscrit dans les principes mêmes de l'État de droit et de la démocratie moderne. Mais, en même temps, ces spécificités ne doivent pas fonder une identité politique particulière, reconnue en tant que telle à l'intérieur de l'espace public, qui doit rester le lieu de l'espace commun, des valeurs, des pratiques et de la langue de la citoyenneté.

Aujourd'hui, les penseurs d'un communautarisme modéré ou contrôlé et les républicains sensibles aux valeurs libérales visent à théoriser les manières de prendre en compte l'ambition proprement démocratique de donner à chacun les moyens de développer toutes ses capacités, de se révéler à lui-même et aux autres dans sa pleine authenticité. Mais comment inscrire à la fois l'égalité du citoyen et la reconnaissance de la diversité des cultures dans des institutions politiques? Même si l'identité de l'individu est effectivement indissociable d'identités collectives, il n'en découle pas que ces identités puissent ou doivent être publiquement reconnues. Il importe en effet de traiter de ces problèmes non en termes philosophiques ou sentimentaux, mais en termes d'institutions sociales et politiques et de se demander concrètement comment faire pour concilier institutionnellement les droits-libertés communs à tous les citoyens, qui constituent un impératif absolu, et les aspirations identitaires légitimes des individus démocratiques.

Quelles que soient les conditions posées à la reconnaissance de droits culturels, la consécration juridique des particularismes comporte en effet le risque d'entraîner dans une logique de revendications sans fin. Au nom de quoi reconnaître dans l'espace public l'usage de telle ou telle langue, l'arabe plutôt que le chinois, l'une des branches du breton et pas les autres? Pourquoi reconnaitre les droits culturels de certains groupes historiques et pas d'autres? Au nom de quels critères de justice? La logique du particularisme a pour fin ultime l'individu. S'il est consacré par l'institution de «droits culturels ", donc politiques, le pluralisme de la vie sociale, fait tout à la fois inévitable et souhaitable dans des sociétés démocratiques, risque de déboucher sur l'inégalité des statuts politiques. Le danger devient grand d'accentuer la fragmentation sociale dans les sociétés démocratiques, alors même que la logique économique et marchande s'impose toujours plus aux dépens des liens civiques, alors même que l'individualisme démocratique conduit chacun à cultiver son moi. Jusqu'à présent, nous ne connaissons pas d'exemple de société moderne dans laquelle la reconnaissance institutionnelle du pluralisme culturel n'a pas conduit au pluralisme social et politique.

Dès lors que les valeurs démocratiques favorisent la tolérance à l'égard de toutes les recherches de l'authenticité de chacun et de tous, dès lors que les individus démocratiques critiquent les institutions qui géraient les différences et les particularismes par l'espace de la citoyenneté commun à tous, se pose la question de ce qu'on désigne comme le "vivre ensemble", question sociologique par excellence. Accepter toutes les expressions de l'authenticité de chaque individu ou de chaque groupe particulier dans l'espace public comporte le risque de miner les principes de la citoyenneté, qui se définit comme la transcendance des particularismes par les institutions communes. Lorsque les Églises ne contrôlent plus les manifestations du croire et que la République, ou la citoyenneté, est impuissante à imposer la transcendance des particularismes et des identifications ethnico-religieuses émotionnelles, n'assiste-t-on 
pas une «double dérégulation", celle qu'assuraient les Églises, celle que garantissaient les lois de la République ${ }^{23}$ ? Or, les institutions ne sont-elles pas nécessaires pour gérer les tensions inhérentes à l'ordre démocratique et protéger les plus faibles?

41 La double dérégulation analysée par Danièle Hervieu-Léger, celle des institutions religieuses et celle des institutions politiques, ne constitue-t-elle pas un risque pour le projet démocratique lui-même et pour le destin des démocraties? Car, il est vrai qu'« on ne rencontrera jamais, quoi qu'on fasse, de véritable puissance parmi les hommes, que dans le cours libre des volontés. Or, il n'y a au monde que le patriotisme, ou la religion, qui puisse faire marcher pendant longtemps vers un même but l'universalité des citoyens ${ }^{24}$.

\section{NOTES}

1. Ce texte a été écrit à l'occasion d'une rencontre organisée à Jérusalem en hommage à Shmuel Eisenstadt à l'occasion de son $80^{\circ}$ anniversaire. Shmuel Eisenstadt est l'un de ceux qui font honneur à notre discipline.

2. Dominique Schnapper, « Le sens de l'ethnico-religieux », Archives de Sciences Sociales des Religions, 81, janvier-mars 1993, p. 149-163.

3. Andrew M. GREELEY, Ethnicity in the United States. A Preliminary Reconnaissance, New York, Wiley, 1972, p. 27.

4. Sur la justification de ce terme voir Schnapper, op. cit., 1993.

5. Les paragraphes qui suivent résument le type idéal élaboré dans Dominique Schnapper, La communauté des citoyens. Sur l'idée moderne de nation, Paris, Gallimard, 1994, réédition «folio-actuel », 2003 (tr. américaine, Transaction, 1998).

6. Qui se traduit concrètement dans une série de liens sociaux spécifiques mais qui prennent leur sens à l'intérieur de ce lien politique fondateur.

7. Sur la justification de ce concept, voir Dominique Schnapper, La relation à l'autre. Au cœur de la pensée sociologique, Paris, Gallimard, 1998, p. 75 (coll. « nrf/essais »).

8. François Luchaire, cité dans Claude Emeri, Jacques Zylberberg, dirs., Citoyenneté et nationalité, perspectives en France et au Québec, Paris, PUF, 1991, p. 135.

9. Ce thème est développé dans Schnapper, op. cit., 1994.

10. Louis Dumont, L'idéologie allemande. France-Allemagne et retour, Paris, Gallimard, 1991 (coll. «Bibliothèque des sciences humaines ».

11. Tzvetan Todorov, Nous et les autres, La question de l'autre, Paris, Seuil, 1989, p. 436.

12. Louis Dumont, Homo hierarchicus, Paris, Gallimard, 1966, annexe A (écrit en 1960) (coll. «Bibliothèque des sciences humaines »).

13. Pierre Rosanvallon, Le sacre du citoyen, Paris, Gallimard, 1992 (coll. « Bibliothèque des hitoires »).

14. On en trouvera une bonne présentation dans Luc Ferry, Le nouvel ordre écologique. L'arbre, l'animal et l'homme, Paris, Grasset, 1992.

15. Takis Fotopoulos, Vers une démocratie générale. Une démocratie directe, économique, écologique et sociale, Paris, Seuil, 2002, p. 13-14;189. D'ailleurs. « Régénérer la tradition 
démocratique suppose de rompre avec le renoncement postmoderne à tout projet politique universaliste, au nom d'une célébration pseudo-pluraliste de la "différence" et de l'identité", qui n'en accepte pas moins comme naturelles les actuelles institutions universelles de concentration du pouvoir politique et économique - la "démocratie" représentative et l'économie de marché »(p.8).

16. Le phénomène touche tous les pays européens, mais c'est en France qu'il est le plus développé et le plus théorisé.

17. Pour un développement de ces analyses, voir Dominique Schnapper, La démocratie providentielle. Essai sur l'égalité contemporaine, Paris, Gallimard, 2002.

18. Le terme de bien-être étant désormais quelque peu galvaudé par la fréquence de son usage, celui de mieux-être se répand aussi bien dans les hebdomadaires généraux (Le nouvel Observateur, janvier 2003, $\mathrm{n}^{\circ}$ 2044) que dans les innombrables publications spécialisées consacrées à la santé physique et psychique, dont les tirages peuvent faire rêver les auteurs de sciences humaines.

19. L'écrivain soviétique, Boukovski, en arrivant en Occident, avait avancé dans un entretien que la société démocratique libérale était une société d'adolescents cherchant à cumuler des avantages contradictoires.

20. Alexis de Tocqueville, La démocratie en Amérique, Paris, 180, tome 3, $2^{\mathrm{e}}$ partie, chap. 12. 21. Voir, par exemple, Françoise Champion, Danièle Hervieu-Léger, dirs., De l'émotion en religion. Renouveaux et traditions, Paris, Centurion, 1990.

22. François de Singly, Les uns avec les autres. Quand l'individualisme crée du lien, Paris, Armand Colin, 2003.

23. Danièle Hervieu-Léger, « "Renouveaux" religieux et nationalistes : la double dérégulation », in Pierre Birnbaum, dir., Sociologie des nationalismes, Paris, PUF, 1997, p. 163-185.

24. Alexis de Tocqueville, La démocratie.., op. cit., tome premier, chap. 5.

\section{RÉSUMÉS}

On peut interpréter les renouveaux ethniques et religieux à partir des caractéristiques de la "démocratie providentielle ». Ces renouveaux ne sont pas seulement dus au besoin des hommes vivant dans une société rationalisée de donner un sens au mal et au malheur. Ils tiennent aussi à l'ambition utopique ou prométhéenne de la démocratie. L'individu devient premier, l'identification aux particularismes tend à primer sur l'aspiration à l'universel, l'expression des émotions est valorisée aux dépens du contrôle de soi, les institutions de l'Église et de la démocratie représentative sont affaiblies par la critique systématique ou radicale. L'individucitoyen, parce qu'il est la source de la légitimité politique, refuse que la tradition fonde la légitimité des institutions ecclésiales et politiques. Il s'accorde à lui-même le droit absolu de critiquer toutes les institutions en tant que telles. Or, les institutions ne sont-elles pas nécessaires pour gérer les tensions inhérentes à l'ordre démocratique et protéger les plus faibles ? N'existe-til pas là un risque pour le projet démocratique lui-même et pour le destin des démocraties?

Ethnic and religious revivals can be interpreted within the framework of "providential democracies". Such revivals are not due solely to the need people living in rationalized societies 
have to find meaning to evil and unhappiness. They are also linked to the utopian or promethean ambitions of democracy. The individual is foremost; particular identities tend to be more important than universalistic aspirations ; priority is given to the expression of emotions over self-control ; the institutions of the Church and representative democracy are weakened by a systematic or radical criticism. Being the source of political legitimacy, the individual citizen rejects the fact that the legitimacy of ecclesiastical and political institutions should be founded on tradition. He grants himself the absolute right to criticize all institutions as such. Yet, are institutions not necessary to manage the tensions inherent to any democratic order and to protect the weakest? Is there not a risk for the democratic project as such and for the future of democracies?

Es posible interpretar las renovaciones étnicas y religiosas a partir de las características de la "democracia providencial". Estas renovaciones no se deben solamente a la necesidad de los hombres que viven en una sociedad racionalizada de dar un sentido al mal y a la desgracia. Dependen también de la ambición utópica o "prometeana" de la democracia. El individuo se ubica en primer lugar, la identificación con los particularismos tiende a primar sobre la aspiración a lo universal, la expresión de las emociones es valorizada sobre el control de sí, las instituciones de la Iglesia y de la democracia representativa se debilitan por la crítica sistemática o radical. El individuo- ciudadano, en tanto que fuente de legitimidad política, rechaza el hecho de que la tradición funde la legitimidad de las instituciones eclesiales y políticas. Éste se otorga a sí mismo el derecho absoluto de criticar todas las instituciones en tanto que tales. Ahora bien, $¿$ no son las instituciones necesarias para manejar las tensiones inherentes al orden democrático y proteger a los más débiles ? ¿ No existe aquí un riesgo para el proyecto democrático mismo y para el destino de las democracias?

\section{AUTEUR}

\section{DOMINIQUE SCHNAPPER}

École des Hautes Études en Sciences Sociales 